\title{
FENOLOGÍA, PRODUCCIÓN Y VALOR NUTRITIVO DEL PASTO ALPISTE (phalaris arundinacea) EN LA ZONA ALTA LECHERA DE COSTA RICA
}

\author{
Luis Villalobos*
}

Palabras clave: Producción de materia seca, estado fenológico, pastos clima templado, Phalaris arundinacea. Keywords: Dry matter yield, phenological stage, utilization, temperate climate grasses, Phalaris arundinacea.

Recibido: 27/09/11

\section{RESUMEN}

Se evaluó la producción de biomasa, el valor nutricional y la fenología del pasto alpiste (Phalaris arundinacea) con 3 edades de cosecha (49, 70 y 91 días), a lo largo de año y medio, en una finca comercial de ganado lechero ubicada en Santa Rosa de Oreamuno, provincia de Cartago. La producción de biomasa promedio para el pasto alpiste fue $3101 \mathrm{~kg} \cdot \mathrm{ha}^{-1} \cdot$ corte $^{-1}$ de MS. La composición nutricional promedio fue de 17,77\% PC, 55,89\% FDN, 35,93\% FDA, 4,14\% lignina y $66,87 \%$ DIVMS y su contenido energético expresado como TND, ED, EM, $\mathrm{EN}_{\mathrm{L}}(3 \mathrm{X})$ y $\mathrm{EN}_{\mathrm{G}}$ fue $63,07 \%, 2,76 ; 2,13 ; 1,31$ y 0,81 Mcal. $\mathrm{kg}^{-1}$ de MS, respectivamente. Se comparó el fraccionamiento de la PC de los pastos estrella africana (Cynodon nlemfuensis) y ryegrass perenne (Lolium perenne) con la del pasto alpiste, con el que las especies de clima templado presentan un aporte principalmente de la fracción insoluble degradable $\left(\mathrm{B}_{2}+\mathrm{B}_{3}\right)$, mientras el estrella africana lo hace a nivel de la fracción degradable $\left(\mathrm{A}+\mathrm{B}_{1}\right)$. La edad fenológica promedio del pasto alpiste fue 5,96 hojas verdes por rebrote y su edad debe encontrarse entre 5 y 6 hojas para optimizar su producción de biomasa y valor nutricional. El pasto alpiste se ha utilizado en diversas fincas de ganado de leche y ha adquirido importancia como una opción que puede soportar las

Centro de Investigaciones en Nutrición Animal y Escuela de Zootecnia, Universidad de Costa Rica.
Aceptado: 11/01/12

\begin{abstract}
Phenology, yield and nutritional value of reed canary grass (Phalaris arundinacea) in dairy farms in the highlands of Costa Rica. Dry matter yield, nutritional value and phenology of reed canary grass (Phalaris arundinacea) were evaluated with three harvest ages (49, 70 and 91 days), during a year and a half, in a commercial dairy farm in the district of Santa Rosa de Oreamuno, Cartago Province. Average dry-matter yield was $3101 \mathrm{~kg} \cdot \mathrm{ha}^{-1} \cdot$ regrowth $^{-1}$ period. Average nutritional value was $17.77 \%$ $\mathrm{CP}, 55.89 \% \mathrm{NDF}, 35.93 \% \mathrm{ADF}, 4.14 \%$ lignin and $66.87 \%$ IVDMD on dry-matter basis. Estimated energy content expressed as TDN, DE, ME, $\mathrm{NE}_{\mathrm{L}}(3 \mathrm{X})$ and $\mathrm{NE}_{\mathrm{G}}$ was $63.07 \%, 2.76,2.13$, 1.31 and 0.81 Mcal. $\mathrm{kg}^{-1}$ of $\mathrm{DM}$, respectively. $\mathrm{CP}$ fractioning of African star grass (Cynodon nlemfuensis) and perennial ryegrass (Lolium perenne), were compared with that of reed canary grass; the main contribution was obtained from the insoluble degradable fraction $\left(\mathrm{B}_{2}+\mathrm{B}_{3}\right)$ in the temperate species, while african star grass had a higher contribution from the degradable fraction $\left(A+B_{1}\right)$. Average phenological stage of reed canary grass was 5.96 green leaves per regrowth and its leaf stage must be 5 to 6 leaves to optimize dry matter yield and nutritional value. Reed canary grass has been used in several dairy
\end{abstract}

San José, Costa Rica. Correo electrónico: luis.villalobosvillalobos@ucr.ac.cr 
condiciones climatológicas de las regiones altas de Costa Rica; asimismo, ya hay empresas que importan semilla sexual de dicha especie.

\section{INTRODUCCIÓN}

El género Phalaris comprende alrededor de 15 especies de pastos anuales y perennes nativos de la zona templada del Norte con unos pocos en Suramérica, asimismo pertenece a la subfamilia Pooideae y se considera relacionado de forma cercana con Hierochloë del cual difiere en que sus flores de reducido tamaño son más bajas (Pohl 1980). Dos de las principales especies pertenecientes a este género son el Phalaris canariensis y el Phalaris arundinacea, el primero es el "alpiste" utilizado comúnmente en alimentación de aves y el segundo es el pasto sobre el cual se basó la presente investigación y el cual es conocido como pasto alpiste.

De acuerdo con Pohl (1980) el Phalaris arundinacea es una forma estéril del "reed canary grass", dicho autor es quién lo reporta por primera vez en Costa Rica como ornamental en Los Yoses, Cantón de Montes de Oca. En el 2007 se identificó su presencia en la finca del presente estudio, gracias al interés mostrado por el dueño de la finca por medio de la recolección de inflorescencias y su posterior identificación en el herbario de la Escuela de Biología de la Universidad de Costa Rica.

El pasto alpiste se ha utilizado bajo un sistema de corte y acarreo en dicha finca y se menciona por parte del dueño como causa probable de su aparición el arrastre de semillas proveniente de fincas vecinas por lixiviación, asimismo ha mostrado ser agresivo ya que ha desplazado al pasto kikuyo (Kikuyuocloa clandestina) que era la especie predominante en los potreros (Coto A. 2007. Comunicación personal). farms and has gained importance as an option that can withstand the weather conditions in the highlands of Costa Rica; olso, there are already companies importing sexual seed of this species.

De acuerdo con Chalupa et al. (1961) el pasto alpiste, es una especie altamente adaptable bajo diversas condiciones, es comúnmente encontrado en zonas con altas precipitaciones donde el suelo permanece húmedo la mayor parte del año. Debido a esto su crecimiento y producción está altamente relacionado con la distribución de las lluvias pues ha llegado incluso a un estado de latencia en períodos de poca precipitación y persistencia de hasta 5 años sin necesidad de resiembra en clima templado (Clark y Pohl 1996).

Uno de los principales usos que se le ha dado al pasto alpiste es para la producción de heno, sin embargo también se ha utilizado bajo sistemas de pastoreo con bovinos de leche y carne y bajo sistemas de corte y acarreo en ovejas y cabras (Chalupa et al. 1961, Archibald et al. 1962).

El manejo agronómico de las pasturas debe buscar el momento óptimo de cosecha para asegurar un material de alto valor nutricional que sea aprovechado por los hatos de alto potencial productivo (Cherney et al. 1993), con especies de clima templado se han desarrollado metodologías relacionadas con la fenología de la planta y los cambios relativos a nivel nutricional (Fulkerson y Donaghy 2001). Dichas metodologías han sido empleadas en condiciones tropicales de altura con dichas especies y han probado ser una buena herramienta para evaluar el momento óptimo de pastoreo (Villalobos y Sánchez 2010a, Andrade 2006).

El objetivo de la presente investigación fue estimar la producción de biomasa, el valor nutricional y la edad fenológica del pasto $\mathrm{Pha}$ laris arundinacea bajo un sistema de corte con 3 edades de cosecha en una finca productora 
de leche ubicada en la zona de influencia del Volcán Irazú.

\section{MATERIALES Y MÉTODOS}

El presente estudio se realizó en una finca comercial productora de leche ubicada en el distrito de Santa Rosa, Oreamuno, Cartago, ubicado en la sección Oriental del Valle Central (latitud 0954', longitud 8349', altitud 1976 msnm) a una altitud de 2000 msnm. Dicha zona tiene influencia climática del Caribe y se clasifica como bosque húmedo tropical premontano (Holdridge 1947, citado por Janzen 1991), su precipitación promedio anual es de $2239 \mathrm{~mm}$, la temperatura mínima y máxima promedio son $13,4^{\circ} \mathrm{C}$ y $23,3^{\circ} \mathrm{C}$ respectivamente y presentan pocas variaciones durante el año, el brillo solar tiene en promedio 5,5 h.día ${ }^{-1}$ y la velocidad promedio del viento 24,4 $\mathrm{km} \cdot \mathrm{h}^{-1}$ (Retana 2011).

Se realizó un análisis de suelo en las parcelas y se encontró contenidos minerales propios para un suelo de origen volcánico (andisol), las bases mostraron valores medios a bajos $(4,26$; 0,66 y $0,11 \mathrm{cmol}(+) \cdot \mathrm{ml}^{-1}$ de $\mathrm{Ca}, \mathrm{Mg}$ y K respectivamente) pero la acidez y el $\mathrm{pH}$ fueron normales sin llegar a valores encima de los recomendados (Bertsch 1998).

El terreno donde se realizó el estudio se encontraba previamente establecido con el pasto alpiste que invadió de forma gradual espacios previamente ocupados por el pasto kikuyo principalmente (Coto A. 2007. Comunicación personal). Debido a que el terreno escogido para el desarrollo del presente estudio contaba con fertilización rutinaria, las evaluaciones se hicieron sin tomar en cuenta fertilización por lo cual los resultados presentados sirven como base para ampliar conocimientos que incluyen el efecto de la fertilización sobre el pasto en un estudio posterior; además se debió realizar un corte de uniformización de las parcelas experimentales. El terreno utilizado es bordeado de forma cercana por un canal de riego de purines provenientes del corral de estabulación y la sala de ordeño lo cual, aunque no es dirigido al terreno donde se ubicaron las parcelas, si reciben un aporte importante en la época semi-lluviosa.

Se contó con 3 tratamientos experimentales que consistieron en las edades de cosecha evaluadas ( $\mathrm{T} 1=49, \mathrm{~T} 2=70$ y T3=91 días) cada una con 4 repeticiones para un total de 12 parcelas experimentales asignadas aleatoriamente cada una con un área promedio de 9,6 $\mathrm{m}^{2}$. La escogencia de las edades de cosecha se basó en la edad máxima a la que se utiliza el pasto en la finca y de ahí se tomó 2 edades menores para evaluar la conveniencia de uso a diferentes períodos de recuperación; el T2 es cercano a la edad en que se cosecha el pasto normalmente en la finca.

Se realizaron 6 muestreos por tratamiento a lo largo de año y medio (2008 y mitad del 2009). En cada una de las parcelas se cosechó todo el material disponible, se pesó en fresco para obtener el rendimiento por área y de éste se tomó una muestra representativa por medio de la técnica de cuarteo para realizar los análisis nutricionales.

Se determinó el contenido de materia seca (MS) y proteína cruda (PC) (AOAC 1990), extracto etéreo (EE) y cenizas (AOAC 2000), las fracciones de la pared celular (FDN, FDA, hemicelulosa, celulosa y lignina) y la digestibilidad "in vitro" de la materia seca (DIVMS) (Van Soest y Robertson 1985). Se determinó el fraccionamiento de la proteína del pasto alpiste, estrella africana (Cynodon nlemfuensis) y ryegrass perenne (Lolium perenne) con la finalidad de comparar sus diferentes fracciones con base en el procedimiento descrito por Licitra et al. (1996). La energía con sus diferentes expresiones (TND, $\mathrm{ED}, \mathrm{EM}, \mathrm{EN}_{\mathrm{L}} 3 \mathrm{X}$ y $\mathrm{EN}_{\mathrm{G}}$ ) se estimó al emplear las metodologías descritas en el NRC (2001). Las muestras de pasto estrella africana fueron cosechadas por medio de la simulación del pastoreo (a una altura de $10 \mathrm{~cm}$ sobre el suelo) en fincas ubicadas en la zona de Monteverde, Puntarenas y que manejan programas de fertilización establecidos con dosis anuales de $250 \mathrm{~kg}^{-h a^{-1}}$ de N, 113 kg.ha ${ }^{-1}$ de $\mathrm{P}_{2} \mathrm{O}_{5}, 10$ kg.ha ${ }^{-1}$ de $\mathrm{K}_{2} \mathrm{O}, 17 \mathrm{~kg} \cdot \mathrm{ha}^{-1}$ de $\mathrm{MgO}, 12 \mathrm{~kg}^{-h^{-1}}$ de $\mathrm{S}, 10 \mathrm{~kg} \cdot \mathrm{ha}^{-1}$ de $\mathrm{CaO}$ y 0,72 kg.ha-1 de Zn. 
La edad fenológica del pasto alpiste se evaluó por medio del conteo del número de hojas vivas con 10 observaciones por parcela (40/tratamiento) para un total de 72 evaluaciones realizadas ( $n=720$ observaciones).

La información obtenida para las variables dependientes producción de biomasa, calidad nutricional y edad fenológica se sometió a un análisis de regresión lineal siguiente modelo con el objeto de buscar efectos significativos $(p \leq 0,05)$ :

Variables analizadas $=\mu+\mathrm{Edad}_{\mathrm{i}}+\mathrm{e}_{\mathrm{i}}$ $\mu=$ media de las variables estudiadas

Edad=i-ésimo efecto de las edades de cosecha $\mathrm{e}=$ residuales del modelo

Se aplicó la prueba de Duncan para establecer diferencias significativas $(p \leq 0,05)$ entre medias en las edades de cosecha para todas las variables.

\section{RESULTADOS Y DISCUSIÓN}

\section{Producción de biomasa}

La producción de biomasa mostró diferencias altamente significativas por resultado de la edad de cosecha ( $\mathrm{p} \leq 0,0001)$, ya que al incrementar los días de recuperación del pasto, su producción fue mayor (Cuadro 1). La producción de biomasa promedio fue de $3101 \mathrm{~kg} \cdot \mathrm{ha}^{-1}$ de MS, valor menor al encontrado por Villalobos y Sánchez (2010a) y Andrade (2006) para los pastos ryegrass y kikuyo con 4110 y $7238 \mathrm{~kg} \cdot \mathrm{ha}^{-1}$ de MS respectivamente en pastoreo. Araya y Boschini (2005) encontraron valores promedio entre 4658 a 15262 kg.ha- ${ }^{-1}$ de MS en 5 cultivares de Pennisetum purpureum cosechados con edades entre 70 y 140 días de rebrote.

Cuadro 1. Producción de biomasa del pasto Phalaris arundinacea con 3 edades de cosecha en el distrito de Santa Rosa de Oreamuno, Cartago.

\begin{tabular}{|c|c|c|c|c|}
\hline $\begin{array}{c}\text { Edad de } \\
\text { cosecha (días) }\end{array}$ & $\begin{array}{l}\text { Cosechas } \\
\text { anuales }\end{array}$ & $\begin{array}{l}\text { Producción de biomasa } \\
\left(\mathrm{kg} \cdot \mathrm{ha}^{-1} \cdot \operatorname{cosecha}^{-1} \text { de MS }\right)\end{array}$ & $\begin{array}{l}\text { Producción de biomasa anualizada } \\
\left(\mathrm{kg} \cdot \mathrm{ha}^{-1} \cdot \mathrm{anno}^{-1} \text { de MS }\right)\end{array}$ & $\begin{array}{c}\text { Carga animal } \\
\text { estable } \\
\left(\text { U.A.ha }^{-1}\right)\end{array}$ \\
\hline 49 & 7,45 & $2035,50^{\mathrm{b}}$ & 15162 & 4,15 \\
\hline 70 & 5,21 & $3453,70^{\mathrm{a}}$ & 18009 & 4,93 \\
\hline 91 & 4,01 & $3847,60^{\mathrm{a}}$ & 15433 & 4,27 \\
\hline Promedio $^{1}$ & 5,56 & 3101,91 & 16201 & 4,45 \\
\hline
\end{tabular}

a,b medias con letras diferentes en una misma columna difieren entre sí, según la prueba de Duncan (p<0,05).

1 Valores corresponden al promedio de 48 muestras.

La producción anual permite determinar la carga animal que un pasto puede soportar ya sea en pastoreo o en corte; en el caso del pasto alpiste se pudo observar que con edades de 91 días su producción promedio fue mayor, sin embargo con edades de 70 días se obtienen más cosechas anualmente y finalmente repercute en una producción anual mayor (Figura 1).

De acuerdo con la definición de Mandaluniz et al. (2005) de que una unidad animal, equivalente a una vaca de $500 \mathrm{~kg}$ no gestante, debe consumir $10 \mathrm{~kg}$.día ${ }^{-1}$ de MS, se estimó la carga animal estable que podría soportar el pasto

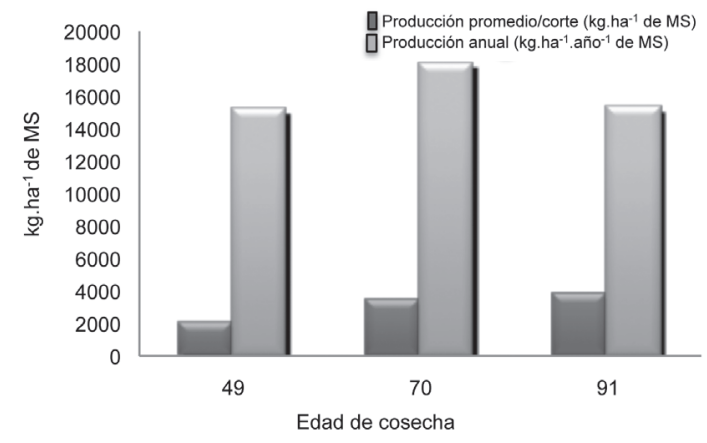

Fig. 1. Producción de biomasa del pasto Phalaris arundinacea con 3 edades de cosecha en el distrito de Santa Rosa de Oreamuno, Cartago. 
alpiste bajo un sistema de corte y acarreo y se puede ver como el T2 es el que puede soportar una mayor carga animal debido a que se pueden obtener más cosechas anualmente en comparación con el T3 (Cuadro 1). Con base en la estimación de la capacidad de carga, se podría decir que la biomasa adicional que produce el pasto alpiste cosechado con edades de rebrote superiores a 70 días, no se justifica pues no permite mantener una carga animal superior durante el año.

\section{Valor nutricional}

El contenido de MS promedio del pasto alpiste fue de $17,20 \%$ y no mostró diferencias significativas entre las diferentes edades de cosecha
(Cuadro 2). Araya y Boschini (2005) encontraron en cultivares de Pennisetum purpureum sometidos a corte valores entre 17,17 a $21,04 \%$ de MS mientras que Villalobos y Sánchez (2010b) encontraron en el pasto ryegrass perenne bajo pastoreo un valor promedio de $15,92 \%$ de MS, por lo tanto se puede ver que el pasto alpiste presenta valores intermedios entre lo que normalmente se puede encontrar en especies comúnmente utilizadas para corte y levemente superiores a las de especies de pasto de piso. Chacón y Vargas (2010) encontraron en pasto Pennisetum purpureum cv. king grass cosechado de 60 a 90 días valores entre 13,03 a $14,43 \%$ MS, lo cual es inferior a los valores encontrados para el pasto alpiste.

Cuadro 2. Contenido de humedad, materia seca, proteína cruda, extracto etéreo y cenizas del pasto Phalaris arundinacea con 3 edades de cosecha en el distrito de Santa Rosa de Oreamuno, Cartago.

\begin{tabular}{lccccc}
\hline Edad (días) & Humedad $(\%)$ & Materia seca $(\%)$ & Proteína cruda $(\%)$ & Extracto etéreo (\%) & Cenizas $(\%)$ \\
\hline 49 & 82,12 & 17,88 & $19,47^{\mathrm{a}}$ & 3,63 & 11,06 \\
70 & 83,32 & 16,68 & $17,14^{\mathrm{b}}$ & 3,37 & 10,60 \\
91 & 82,98 & 17,02 & $16,65^{\mathrm{b}}$ & 3,34 & 10,39 \\
Promedio $^{1}$ & 82,80 & 17,20 & 17,77 & 3,47 & 10,75 \\
\hline
\end{tabular}

a,b medias con letras diferentes en una misma columna difieren entre sí, según la prueba de Duncan ( $\mathrm{p} \leq 0,05)$.

1 Valores corresponden al promedio de 48 muestras.

El manejo que se le ha dado al pasto alpiste en la finca donde se realizó el estudio, comprende un proceso de marchitamiento leve, de tal forma que tenga una mayor concentración de la MS. En los sistemas de corte-acarreo utilizados en las fincas normalmente se debe organizar las labores de tal forma que los animales tengan una ración lo más constante posible que no afecte el rendimiento productivo pues cualquier cambio por pequeño que parezca puede afectar el consumo del grupo y, por tanto, su productividad.

El contenido de PC promedio del pasto alpiste fue $17,77 \%$ y se vio afectado de forma significativa $(\mathrm{p} \leq 0,0001)$ por la edad de cosecha en donde el T1 mostró el contenido proteico mayor. Araya y Boschini (2005) encontraron para cultivares de Pennisetum purpureum contenidos de
PC entre 9,08 y $11,14 \%$, valores que son cercanos a la mitad de los encontrados en el pasto alpiste; por su parte Chacón y Vargas (2010) encontraron una leve disminución en el contenido de PC del pasto king grass de 9,56 a $8,42 \%$ al aumentar la edad de corta de 60 a 90 días.

Los pastos de piso de clima templado $\left(\mathrm{C}_{3}\right)$ pueden alcanzar contenidos de PC mayores al $20 \%$ tal como sucedió con el pasto ryegrass perenne que ha mostrado valores promedio de $25,21 \%$ con periodos de recuperación promedio de 37 días (Villalobos y Sánchez 2010b). En el caso del pasto kikuyo, Andrade (2006) encontró un valor promedio de $22,38 \%$ PC con una edad de cosecha de 28 días promedio. El pasto alpiste es una especie de pasto de clima templado que se utiliza bajo un sistema de corte-acarreo y se 
puede ver que a pesar de manejarse con edades de cosecha superiores a las edades manejadas en pastoreo, su contenido de PC disminuye menos de $2 \%$. Maya et al. (2005) encontraron en el pasto estrella solo y en asociación con Leucaena leucocephala, una marcada tendencia a disminuir el contenido de PC conforme se aumentó la edad de corte.

En la Figura 2 se muestra la extracción de nitrógeno del pasto alpiste anualmente bajo las 3 edades de cosecha, se puede observar que el T2 es el que más extrae debido a su mayor producción de biomasa y su contenido proteico promedio que llegó a valores superiores a los $400 \mathrm{~kg} \cdot \mathrm{ha}^{-1} \cdot \mathrm{año}^{-1}$ de N. Chalupa et al. (1961) analizaron el efecto de la fertilización nitrogenada sobre el valor nutricional del pasto alpiste para la producción

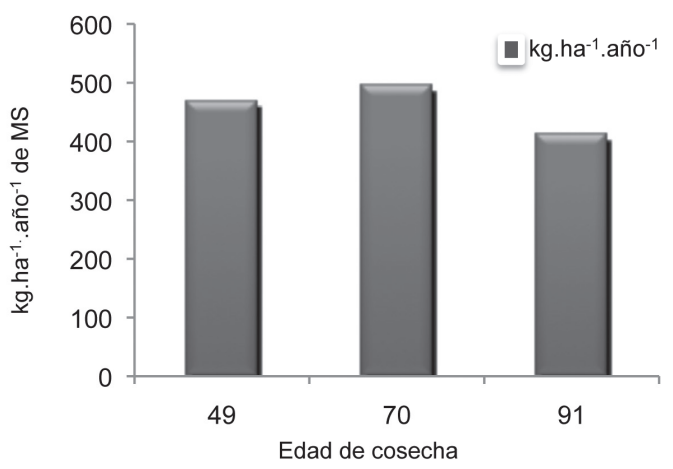

Fig. 2. Extracción de nitrógeno del pasto Phalaris arundinacea con 3 edades de cosecha en el distrito de Santa Rosa de Oreamuno, Cartago. de heno y el balance de $\mathrm{N}$ en novillas Holstein alimentadas con dicho forraje, y se encontró que al incrementar la dosis de fertilización de 0 a 114 y $227 \mathrm{~kg} \cdot \mathrm{ha}^{-1} \cdot \mathrm{año} \mathrm{o}^{-1}$ se incrementó el contenido de proteína (PC), extracto etéreo (EE) y energía bruta (EB) mientras la fibra cruda (FC) y el extracto libre de nitrógeno (ELN) disminuyeron. Como se puede ver el pasto alpiste tiene una alta capacidad de absorción de $\mathrm{N}$ lo cual permite que el valor nutricional del pasto sea mayor y una alta capacidad de producir biomasa asociada con dicho nutriente.

El contenido promedio de extracto etéreo y cenizas fueron 3,47 y $10,75 \%$ y ambos no mostraron diferencias significativas entre los tratamientos por lo que la edad de corte no afecta dichos componentes en el pasto alpiste (Cuadro 2), el mismo comportamiento fue encontrado por Maya et al. (2005) con el pasto estrella cosechado a 3 diferentes edades, donde el contenido de EE y cenizas no mostró diferencias significativas conforme se incrementó la edad de corte.

De acuerdo con Licitra et al. (1996) la separación de la proteína y las fracciones de nitrógeno usados por el modelo de carbohidratos y proteína neta de la Universidad de Cornell comprende 5 fracciones denotadas como $\mathrm{A}, \mathrm{B}_{1}$, $\mathrm{B}_{2}, \mathrm{~B}_{3}$ y C (Cuadro 3). La fracción A representa la fracción de nitrógeno no proteico mientras que la proteína verdadera se separa en $\mathrm{B}_{1}, \mathrm{~B}_{2}, \mathrm{~B}_{3}$ basadas en su orden decreciente de solubilidad a nivel ruminal y la fracción $\mathrm{C}$ representa la parte

Cuadro 3. Fraccionamiento de la proteína de los pastos estrella africana, ryegrass tetraploide y alpiste forrajero.

\begin{tabular}{|c|c|c|c|c|c|c|}
\hline \multirow{2}{*}{ Pasto } & \multirow{2}{*}{$\begin{array}{l}\mathrm{PC} \\
(\%)\end{array}$} & \multicolumn{5}{|c|}{$\begin{array}{l}\text { Fraccionamiento de la proteína } \\
\qquad(\% \text { de la PC) }\end{array}$} \\
\hline & & A & $\mathrm{B}_{1}$ & $\mathrm{~B}_{2}$ & $\mathrm{~B}_{3}$ & $\mathrm{C}$ \\
\hline Estrella africana ${ }^{2}$ & 20,38 & 7,93 & 1,44 & 2,51 & 5,38 & 3,12 \\
\hline Ryegrass perenne & 20,00 & $\begin{array}{c}(38,52) \\
2,94\end{array}$ & $\begin{array}{c}(7,79) \\
1,54\end{array}$ & $\begin{array}{c}(12,14) \\
6,65\end{array}$ & $\begin{array}{c}(26,71) \\
7,83\end{array}$ & $\begin{array}{r}(14,84) \\
5,29\end{array}$ \\
\hline tetraploide ${ }^{3}$ & 24,25 & $(12,20)$ & $(6,35)$ & $(27,63)$ & $(31,77)$ & $(22,05)$ \\
\hline Alpiste forrajero 4 & 19,28 & $\begin{array}{c}5,03 \\
(25,83) \\
\end{array}$ & $\begin{array}{c}1,52 \\
(7,79)\end{array}$ & $\begin{array}{r}7,57 \\
(39,39) \\
\end{array}$ & $\begin{array}{r}3,69 \\
(19,32) \\
\end{array}$ & $\begin{array}{r}1,47 \\
(7,67) \\
\end{array}$ \\
\hline
\end{tabular}

\footnotetext{
Valores porcentuales de la PC entre paréntesis.

8 muestras 25 días de recuperación.

11 muestras 45 días de recuperación.

16 muestras 70 días de recuperación.
} 
insoluble en solución ácida detergente y que se asume como no digestible por los rumiantes.

En este proyecto de investigación se decidió analizar el fraccionamiento de proteína de una parte de las muestras recolectadas del pasto alpiste con edades de 70 días así como muestras con las que se contaba de custodia de pasto estrella africana y ryegrass perenne tetraploide. De acuerdo con Elizalde et al. (1999), debido a que las fracciones de PC difieren en la tasa y grado de degradación ruminal, las proporciones de cada fracción presentes en los alimentos para animales se considera que influyen sobre las cantidades degradadas en rumen y $\mathrm{PC}$ no aprovechable consumida por el animal.

Se encontró valores para la fracción de nitrógeno no proteico $(\mathrm{NNP}=\mathrm{A})$ entre 12,20$38,52 \%$ de la PC para las 3 especies de pastos (Cuadro 3). WingChing y Rojas (2006) evaluaron el efecto de la fuente y la dosis de fertilizante nitrogenado sobre el fraccionamiento proteico del sorgo negro con cobertura permanente de maní forrajero y encontraron para la fracción A un valor entre 5,76-9,14\% de la PC total, dichos valores son inferiores a las encontrados en la presente investigación. En especies forrajeras de clima templado se han encontrado valores de la fracción A entre 21,8-23,4\% de la PC, con mayor contenido en alfalfa (Medicago sativa) y menor en el pasto festuca (Festuca arundinacea) infectado con endófito (Acremonium coenophialum), dichos valores son muy cercanos entre sí, a diferencia de lo mostrado en la presente investigación (Elizalde et al. 1999).

En el caso del pasto estrella, fue el que presentó el valor de la fracción A mayor, ya que mostró contenidos entre 25-60\% de la PC, esto coincide con lo mostrado por Johnson et al. (2001) con la misma especie con $27,9 \%$ y con el pasto bermuda (Cynodon dactylon) con 31,0\% de la PC; por esto se menciona a las especies de pastos tropicales como capaces de favorecer la producción de proteína microbial si se complementan con una fuente de carbohidratos no fibrosos que sean rápidamente degradables en el rumen.
La fracción $\mathrm{B}_{1}$ mostró valores muy cercanos en las 3 especies y con poca variación $(6,35-7,79 \%$ de la PC), valores similares a los encontrados por WingChing y Rojas (2006) para el sorgo negro con cobertura vegetal $(4,03$ $6,09 \%$ de la PC); mencionan los autores que dicha fracción es poco importante en los forrajes tropicales pues su concentración es menor al $10 \%$ de la PC lo cual coincide con lo encontrado en la presente investigación incluso en 2 especies de pastos de clima templado. Elizalde et al. (1999) encontraron una proporción mayor de la fracción $\mathrm{B}_{1}$ en la alfalfa que en los pastos bromus (Bromus biebersteinii) y festuca libre y con endófito $(17,1 ; 12,1 ; 13,2$ y $14,5 \%$ de la PC respectivamente), lo cual a su vez explicó el contenido mayor de $\mathrm{N}$ soluble o PC degradable en rumen $\left(A+B_{1}\right)$ de la primera.

Los animales rumiantes tienen la capacidad de producir proteína microbial a partir del nitrógeno presente en los alimentos y la determinación del fraccionamiento de la proteína busca facilitar las prácticas de alimentación según el forraje que se utilice, sin embargo el método no es específico ni tiene límites definidos precisamente para las fracciones que se han mencionado, es por esto que se considera que hay fracciones que comparten características y que los animales aprovechan similar. Esto reduce las fracciones de 5 a 3, las cuales son la proteína degradable $\left(\mathrm{A}+\mathrm{B}_{1}\right)$, insoluble aprovechable $\left(\mathrm{B}_{2}+\mathrm{B}_{3}\right)$ e indigerible (C) (Figura 3).

En la Figura 3 se muestra en términos porcentuales las fracciones degradable, insoluble aprovechable e indigerible para las 3 especies de pastos, se puede ver la importancia de la fracción degradable en rumen del pasto estrella con un valor de 46,31\% de la PC. En los pastos ryegrass perenne y alpiste forrajero la fracción insoluble aprovechable es la de mayor importancia. En términos de las prácticas de alimentación, el pasto estrella puede favorecer la producción de proteína microbial y en el caso de los pastos ryegrass perenne y alpiste forrajero su aporte de nutrientes tiene mayor importancia a nivel intestinal por las características de sobrepaso de las fracciones $\mathrm{B}_{2}$ 


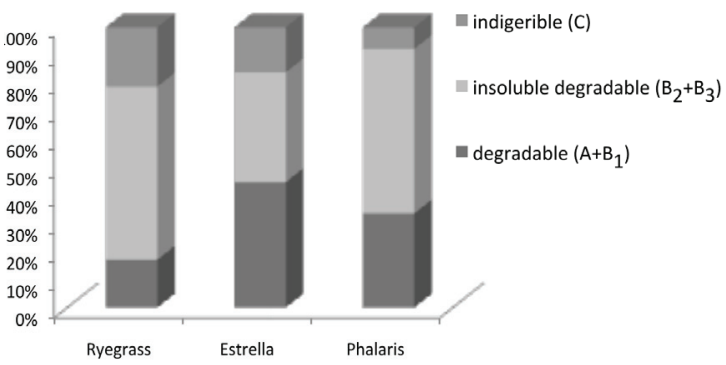

Fig. 3. Fraccionamiento de la proteína cruda de los pastos estrella africana, ryegrass perenne y alpiste forrajero en términos de degradabilidad.

y $\mathrm{B}_{3}$ y por tanto se debe buscar el uso de carbohidratos de más lenta liberación (almidones) en la suplementación pues si se brinda fuentes de carbohidratos rápidamente degradables no serán aprovechados a nivel ruminal para la producción proteica microbial.

Elizalde et al. (1999) para especies de clima templado, encontró valores entre 35,1$40,5 \%$ degradable en rumen de la PC, valores superiores a los del pasto ryegrass perenne y el pasto alpiste con 18,55 y $33,62 \%$ de la PC.

La fracción $\mathrm{B}_{2}$ mostró valores entre 12,14$39,39 \%$ de la PC, los valores menor y mayor fueron de estrella y alpiste forrajero respectivamente y el ryegrass mostró un valor intermedio de $27,63 \%$. El alpiste forrajero tiene potencial de incrementar los rendimientos del animal con su aporte de una parte importante del $\mathrm{N}$ a nivel intestinal (sobrepaso), el pasto estrella por su parte tiene un contenido menor de dicha fracción de proteína verdadera, razón por la cual es importante el uso de fuentes de carbohidratos no fibrosos en la dieta de las vacas en producción para hacer un uso más eficiente del $\mathrm{N}$ soluble en rumen para producir proteína microbial que pase al intestino y sea utilizada por el animal.

La fracción $\mathrm{B}_{2}$ ha mostrado ser la de mayor importancia en especies de clima templado, pues mostró valores de 42,6\% en bromus hasta 51,6\% de la PC en alfalfa (Elizalde et al. 1999). Archibald et al. (1962) encontraron un alto contenido de proteína en heno de pasto alpiste $(21,3 \%)$ y un alto porcentaje mostró ser proteína verdadera
(15,8\% o $74,1 \%$ del total). El contenido tan alto de proteína en el pasto se debió, de acuerdo con los autores, a que el terreno recibía nutrientes por efecto de lixiviación de otros terrenos cercanos.

La fracción $\mathrm{B}_{3}$ mostró un rango menor entre las 3 especies de pastos (19,32-31,77\% de la $\mathrm{PC}$ ), dichos valores son menores en términos porcentuales a los encontrados por WingChing y Rojas (2006) para el sorgo negro forrajero (40,66-43,91\% de la PC) pues esta fracción fue la más importante para dicho pasto, lo mismo sucedió en la presente investigación con el pasto ryegrass perenne con $31,77 \%$ de la PC por lo que se puede ver como las 2 especies de pastos de clima templado tienen una alta proporción de su $\mathrm{N}$ en forma de proteína verdadera a diferencia del pasto estrella en el que la fracción A es la de mayor importancia (Figura 3).

La fracción $\mathrm{C}$ representa el $\mathrm{N}$ no disponible o ligado a la pared celular y que sobrepasa rumen e intestino y por lo tanto no es aprovechable por el animal, en el caso de la presente investigación el rango en las 3 especies fue de 7,67\% para el alpiste forrajero a $22,05 \%$ de la PC para el pasto ryegrass perenne, este último presenta un valor mayor debido a que la edad de cosecha fue mayor (45 días) que la del pasto estrella y alpiste (25 y 70 días respectivamente) lo cual coincide con lo encontrado por Elizalde et al. (1999) donde la fracción $\mathrm{C}$ incrementó conforme aumentaban los días de cosecha pero no se vio afectado por la especie de forraje. WingChing y Rojas (2006) encontraron en el sorgo negro que entre 18,48$18,96 \%$ de la PC lo representa la fracción C, dicha proporción es superior a la hallada por Elizalde et al. (1999) en especies de clima templado (1,0$6,6 \%$ de la PC).

El contenido de FDN promedio en el pasto alpiste fue de 55,89\% y aumentó de forma significativa $(\mathrm{p}<0,05)$ posterior a los 70 días de cosecha (Cuadro 4). Maya et al. (2005) encontraron un aumento en el contenido de FDN del pasto estrella africana que pasó de 65,78 a $76,92 \%$ en edades de 28 y 35 días respectivamente y una leve disminución con 42 días debido a la morfología del pasto que genera rebrotes laterales con hojas 
Cuadro 4. Contenido de los componentes de la pared celular y digestibilidad del pasto alpiste con 3 edades de cosecha en el distrito de Santa Rosa de Oreamuno, Cartago.

\begin{tabular}{ccccccc}
\hline Edad (días) & FDN (\%) & FDA (\%) & Lignina (\%) & Hemicelulosa (\%) & Celulosa (\%) & DIVMS (\%) \\
\hline 49 & $53,18^{\mathrm{b}}$ & 34,60 & 3,78 & 18,79 & 31,04 & $70,55^{\mathrm{a}}$ \\
70 & $57,77^{\mathrm{a}}$ & 36,86 & 4,26 & 20,91 & 32,59 & $65,95^{\mathrm{b}}$ \\
91 & $56,76^{\mathrm{a}}$ & 36,36 & 4,42 & 20,16 & 32,34 & $63,38^{\mathrm{b}}$ \\
Promedio $^{1}$ & 55,89 & 35,93 & 4,14 & 20,01 & 32,01 & 66,87 \\
\hline
\end{tabular}

a,b medias con letras diferentes en una misma columna difieren entre sí, según la prueba de Duncan $(\mathrm{p} \leq 0,05)$.

1 Valores corresponden al promedio de 48 muestras.

nuevas; este mismo comportamiento se puede atribuir al pasto alpiste debido a su alta capacidad de desarrollo lateral, razón que explica su rápido establecimiento en las fincas donde se maneja.

Chacón y Vargas (2010) encontraron que el king grass aumenta su contenido de FDN de 73,78 a $76,91 \%$ con edades de corta de 60 y 90 días respectivamente, dichos valores son superiores a los encontrados en el pasto alpiste, mientras que Villalobos y Sánchez (2010b) encontraron para el pasto ryegrass perenne un valor de FDN promedio de $49,76 \%$ con edades de cosecha máximas de 45 días; este último valor es más cercano a lo encontrado en la presente investigación y coincide con lo encontrado por Elizalde et al. (1999) con valores de FDN entre 50,1-51,2\% para el pasto bromus y festuca.

Se sabe que la fibra de la ración debe ser aprovechable por los microorganismos del rumen y esto es a su vez un indicador del valor nutricional de cualquier forraje, sin embargo en estudios realizados con animales, se encontró una disminución en la digestibilidad aparente de la fibra conforme se aumentó las dosis de fertilización nitrogenada (Chalupa et al. 1961). El contenido de fibra detergente neutro (FDN) de un pasto no debe ser menor de $40 \%$ y no más de $55 \%$ de la MS para facilitar el balance de las raciones del ganado lechero; dicho valor puede ser alcanzado con especies de clima templado a través de un adecuado manejo, en especies de pastos tropicales puede ser más difícil lograrlo debido a su contenido mayor de pared celular como adaptación para el clima caliente del trópico (Cherney et al. 1993). Con base en lo anterior, se puede ver que el pasto alpiste tiene un contenido de FDN que tiene potencial para dar una adecuada respuesta productiva por el ganado de leche que lo consume sin afectar el consumo de MS por efecto de llenado físico.

Los demás componentes de la pared celular mostraron valores promedio de 35,93; 20,01; 32,01 y 4,14\% de la MS para FDA, hemicelulosa, celulosa y lignina respectivamente y no se vieron afectados $(p<0,05)$ por la edad de cosecha. En el pasto ryegrass perenne, Villalobos y Sánchez (2010b) han encontrado valores de FDA de $25,57 \%$, valor que es 10 unidades porcentuales menor que el alpiste, esto a su vez coincide con Elizalde et al. (1999) con valores entre 24,4$26,9 \%$ de la MS.

Al considerar los demás componentes de la FDN en el pasto alpiste, se puede ver como la hemicelulosa mostró valores inferiores a lo reportado para especies de clima templado, mientras que la celulosa por el contrario fue superior en más de 10\% (Villalobos y Sánchez 2010b, Elizalde et al. 1999). La lignina es un constituyente de la pared celular que limita el acceso de los microorganismos del rumen a los nutrientes intracelulares, lo cual se manifiesta en una reducción en su digestibilidad, por lo que en el pasto alpiste a pesar de que su aumento no fue estadísticamente significativo $(\mathrm{p}<0,05)$, podría limitar la digestibilidad de la pared celular si no se manejan adecuadas edades de cosecha. Archibald et al. (1962) encontraron una correlación negativa y altamente significativa $(r=-0,54, \mathrm{p}<0,01)$ entre el contenido de lignina y la digestibilidad de la materia seca en henos de pasto alpiste y alfalfa. 
Chacón y Vargas (2010) analizaron el contenido de la pared celular del king grass y dentro de sus componentes la lignina es fundamental como indicador de digestibilidad para lo cual encontraron valores entre $12,15-13,59 \%$ para las edades de corta de 60 y 90 días respectivamente, dichos valores son superiores a los mostrados por especies de clima templado como el ryegrass perenne $(3,29 \%)$, bromus $(1,69 \%)$, festuca (1,61\%) (Villalobos y Sánchez 2010b, Elizalde et al. 1999).

La digestibilidad in vitro de la materia seca (DIVMS) promedio del pasto alpiste fue $66,87 \%$ y mostró una disminución significativa $(\mathrm{p}<0,05)$ al aumentar la edad de cosecha (Cuadro 4). Maya et al. (2005) analizaron el comportamiento del pasto estrella solo y con Leucaena en asociación a diferentes edades de cosecha y conforme se incrementó los días de rebrote la digestibilidad disminuyó de forma significativa $(\mathrm{p}<0,05)$, efecto asociado principalmente al aumento en el contenido de FDN, comportamiento similar al mostrado en el pasto alpiste donde las edades de cosecha de 70 y 91 días fueron las que mostraron valores inferiores resultado del aumento en pared celular.

El valor de DIVMS del pasto alpiste es inferior al mostrado por especies de pastos de clima templado utilizados en condiciones tropicales de altura como el ryegrass perenne con 77,95\% (Villalobos y Sánchez 2010b) y muy cercanos al de especies tropicales de altura como el kikuyo con 65,43\% (Andrade 2006).
El pasto alpiste presentó una disminución en su contenido de energía conforme se incrementó la edad de cosecha (Cuadro 5). Las diferentes fracciones energéticas analizadas mostraron valores promedio de $63,07 \%$ y 2,$76 ; 2,13 ; 1,31$ y 0,81 Mcal. $\mathrm{kg}^{-1}$ de MS para el total de nutrientes digestibles, energía digestible, metabolizable, neta de lactancia y neta de ganancia respectivamente. Chalupa et al. (1961) encontraron valores de TND y ED de 55,91\% y 2,48 Mcal. $\mathrm{kg}^{-1}$ de MS para el pasto alpiste sin recibir fertilización nitrogenada; por su parte Maya et al. (2005) encontraron en el pasto estrella valores de energía metabolizable cada vez menores al incrementar la edad de cosecha de 28 a 42 días, al pasar de 2,22 a 1,92 Mcal. $\mathrm{kg}^{-1}$ de MS, dichos valores son inferiores a los encontrados en la presente investigación para el pasto alpiste, lo cual permite que el ganado lechero tenga un desempeño productivo superior al consumir materiales forrajeros de mayor calidad nutricional.

Villalobos y Sánchez (2010b) encontraron en el pasto ryegrass perenne valores de TND cercanos a los del pasto alpiste $(61,95 \%)$, pero contenido de ED superior (2,92 Mcal. $\mathrm{kg}^{-1}$ de MS), por su parte Andrade (2006) encontró un TND de $63,72 \%$ y ED 2,98 Mcal.kg-1 de MS lo cual permite evidenciar que el pasto alpiste, bajo un sistema de corte-acarreo, tiene un valor nutricional cercano al de los principales pastos de piso utilizados en las lecherías tropicales de altura.

Cuadro 5. Contenido de energía del pasto Phalaris arundinacea con 3 edades de cosecha en el distrito de Santa Rosa de Oreamuno, Cartago.

\begin{tabular}{|c|c|c|c|c|c|}
\hline Edad (días) & $\begin{array}{c}\text { Total de } \\
\text { nutrientes } \\
\text { digestibles (\%) }\end{array}$ & $\begin{array}{l}\text { Energía digestible } \\
\text { (Mcal.kg }{ }^{-1} \text { de MS) }\end{array}$ & 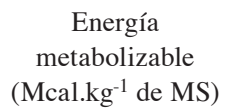 & 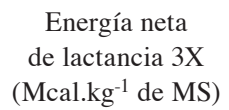 & 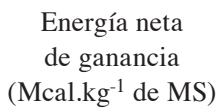 \\
\hline 49 & 64,12 & 2,81 & 2,18 & 1,34 & 0,85 \\
\hline 70 & 62,22 & 2,72 & 2,10 & 1,29 & 0,79 \\
\hline 91 & 62,87 & 2,74 & 2,11 & 1,30 & 0,80 \\
\hline Promedio $^{1}$ & 63,07 & 2,76 & 2,13 & 1,31 & 0,81 \\
\hline
\end{tabular}

1 Valores corresponden al promedio de 48 muestras. 
Los pastos kikuyo y ryegrass han mostrado, en condiciones tropicales de altura, valores de energía metabolizable y neta de lactancia entre 2,37-2,45 y 1,48-1,53 Mcal.kg-1 de MS respectivamente (Andrade 2006, Villalobos y Sánchez 2010b), dichos valores son superiores a los encontrados en el pasto alpiste (2,13 y 1,31 Mcal.kg ${ }^{-1}$ de MS) y su contenido tiende a disminuir conforme se incrementa la edad de cosecha (Cuadro 5). Con base en el contenido energético del pasto alpiste, es conveniente escoger un suplemento nutricional balanceado que pueda aportar los nutrientes limitantes para llenar los requerimientos de los animales en producción (Weiss 2008).

\section{Edad fenológica}

La edad fenológica promedio del pasto alpiste fue 5,96 hojas verdes por rebrote y no se vio afectada de forma significativa $(\mathrm{p}<0,05)$ por los días de cosecha (Cuadro 6 y Figura 4). Se puede ver como la edad fenológica aumenta levemente a los 70 días y vuelve a disminuir a los 91 días, la forma de crecimiento puede ser la causa de dicho comportamiento debido a su capacidad de producir rebrotes laterales que le permiten ampliar su área de cobertura posterior a los 70 días de cosecha.

Con base en lo obtenido en el presente estudio se puede observar que la edad fenológica

Cuadro 6. Edad fenológica del pasto Phalaris arundinacea con 3 edades de cosecha en el distrito de Santa Rosa de Oreamuno, Cartago.

Edad de cosecha (días)

\begin{tabular}{cc}
\hline 9 & 5,8278 \\
70 & 6,0725 \\
91 & 5,9775 \\
Promedio $^{1}$ & 5,9638 \\
\hline
\end{tabular}

Valores corresponden al promedio de 10 observaciones/parcela/muestreo con 4 parcelas por tratamiento y 5 muestreos $(n=600)$.

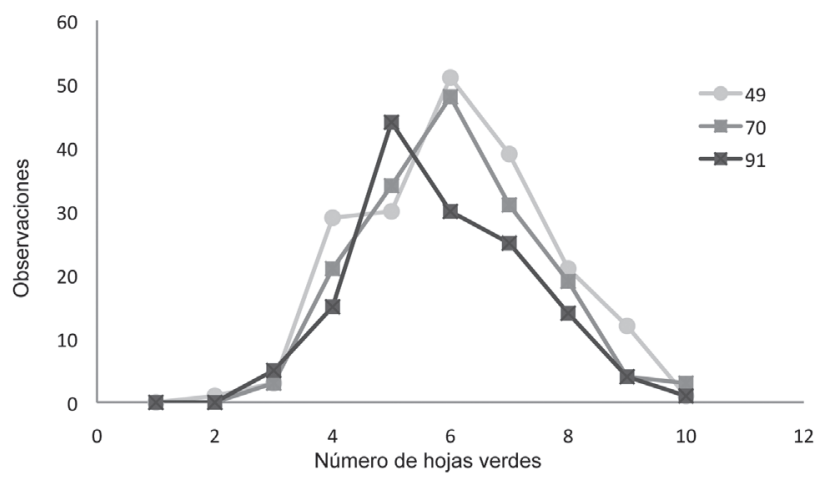

Fig. 4. Distribución de la frecuencia de observaciones del número de hojas verdes por rebrote del pasto Phalaris arundinacea con 3 edades de cosecha en el distrito de Santa Rosa de Oreamuno, Cartago. 
adecuada para el pasto alpiste se encuentra entre 5 y 6 hojas verdes/rebrote (Figura 4) ya que posterior a dicha etapa la planta pierde las primeras hojas en emerger en forma de material senescente y su valor nutricional disminuye.

\section{CONCLUSIONES Y RECOMENDACIONES}

La producción de biomasa del pasto alpiste se optimiza a una edad de 70 días pues se obtienen más cosechas anuales, mayor producción anual y permite soportar una mayor carga animal que en edades de cosecha superiores en donde la cantidad de días de recuperación no compensa la producción marginal de MS obtenida. La producción de biomasa promedio fue de 3101,91 kg.ha-1 de MS.

El contenido de MS se mantuvo relativamente constante en las 3 edades de cosecha, mientras que el contenido de PC disminuyó al aumentar los días de cosecha. El pasto alpiste tiene niveles de extracción de $\mathrm{N}$ del suelo de hasta $400 \mathrm{~kg} \cdot \mathrm{ha}^{-1} \cdot \mathrm{año}^{-1}$, por lo que su utilización en sistemas de corte-acarreo debe ir acompañada idealmente de programas de fertilización con el fin de no degradar el suelo.

El fraccionamiento de la $\mathrm{PC}$ de las 3 especies de pasto analizadas mostró que las especies tropicales como la estrella africana, tienen capacidad de favorecer la producción de proteína microbial a nivel del rumen pues su fracción degradable $\left(\mathrm{A}+\mathrm{B}_{1}\right)$ es la de mayor importancia, mientras que en las especies de clima templado como el alpiste y el ryegrass perenne, la fracción insoluble aprovechable es la que tiene un porcentaje mayor entre todas las fracciones. Las prácticas de alimentación deben buscar el máximo aprovechamiento del $\mathrm{N}$ contenido en la planta, por medio del uso de suplementos adecuados a las condiciones de disponibilidad de los nutrientes a lo largo del tracto gastrointestinal de los animales rumiantes.

Los componentes de la pared celular del pasto alpiste fueron mayores conforme se incrementó la edad de cosecha, lo cual provoca una reducción en la DIVMS y en el contenido de energía.

El pasto alpiste debe utilizarse en una edad fenológica entre 5 y 6 hojas para obtener una adecuada producción de biomasa, y un valor nutricional que permita su utilización en sistemas intensivos de producción láctea en las zonas altas de Costa Rica.

\section{AGRADECIMIENTOS}

Al propietario de la finca donde se realizó esta investigación: Ing. Alvaro Coto, así como a los trabajadores de la finca por la colaboración brindada durante el año en que se realizó la investigación.

\section{LITERATURA CITADA}

ANDRADE M. 2006. Evaluación de técnicas de manejo para mejorar la utilización del pasto kikuyo (Pennisetum clandestinum Hochst. Ex chiov) en la producción de ganado lechero en Costa Rica. Tesis de licenciatura, Universidad de Costa Rica, San José, Costa Rica. $225 \mathrm{p}$.

ARAYA M., BOSCHINI C. 2005. Producción de forraje y calidad nutricional de variedades de Pennisetum purpureum en la Meseta Central de Costa Rica. Agronomía Mesoamericana 16(1):37-43.

ARCHIBALD J.G., BARNES H.D., FENNER F., GERSTEN B. 1962. Digestibility of Alfalfa hay and "reed canary grass" hay measured by two procedures. J Dairy Sci 45:858-860.

ASSOCIATION OF OFFICIAL AGRICULTURAL CHEMISTS. 1990. Official methods of analysis. 15 ed. Washington, D.C. 1008 p.

ASSOCIATION OF OFFICIAL AGRICULTURAL CHEMISTS. 2000. Official methods of analysis. 17 ed. Washington, D.C.

BERTSCH F. 1998. La fertilidad de los suelos y su manejo. San José, Costa Rica. Asociación Costarricense de la Ciencia del Suelo. 157 p.

CHACÓN P., VARGAS C. 2009. Digestibilidad y calidad del Pennisetum purpureum cv. king grass a tres edades de rebrote. Agronomía Mesoamericana 20(2):399408.

CHALUPA W.V., CASON J.L., BAUMGARDT B.R. 1961. Nutritive value of "reed canary grass" as hay when grown with various nitrogen levels. J. Dairy Sci. 44:874-878. 
CHERNEY D.J., CHERNEY J.H., LUCEY R.F. 1993. In vitro digestion kinetics and quality of perennial grasses as influenced by forage maturity. J. Dairy Sci. 76:790-797.

CLARK L.G., POHL R.W. 1996. Agnes Chase's First Book of Grasses. The structure of grasses explained for beginners. Smithsonian Institution Press, fourth edition. United States of America. 127 p.

ELIZALDE J.C., MERCHEN N.R., FAULKNER D.B. 1999. Fractionation of fiber and crude protein in fresh forages during the spring growth. Journal of Animal Science 77:476-484.

FULKERSON W.J., DONAGHY D.J. 2001. Plant-soluble carbohydrate reserves and senescence key criteria for developing an effective grazing management system for ryegrass-based pastures: a review. Australian Journal of Experimental Agriculture. 41:261-275.

JANZEN D.H. 1991. Historia Natural de Costa Rica. $1^{\text {a }}$ ed. Editorial de la UCR, San José, Costa Rica. 822 p.

JOHNSON C.R., REILIING B.A., MISLEVY P., HALL M.B. 2001. Effects of nitrogen fertilization and harvest date on yield, digestibility, fiber, and protein fractions of tropical grasses. Journal of Animal Science 79:2439-2448.

LICITRA G., HERNANDEZ T.M., VAN SOEST P.J. 1996. Standardization of procedures for nitrogen fractionation of ruminant feeds. Animal Feed Science Technology 57:347-358.

MANDALUNIZ N., RUIZ R., OREGUI L. 2005. Propuesta de definición de unidad animal y metodología de estimación, para su aplicación en sistemas de pastoreo extensivo, pp 274-280. In: Producciones agroganaderas: Gestión eficiente y conservación del medio natural (Vol. I). SEEP.
MAYA G., DURÁN C., ENRIQUE J. 2005. Valor nutritivo del pasto estrella solo y en asociación con leucaena a diferentes edades de corte durante el año. Acta Agronómica. Vol. 54(2).

NATIONAL RESEARCH COUNCIL. 2001. Nutrient Requirements of Dairy Cattle. $7^{\text {th }}$ rev. Ed Washington, D.C. National Academy Press. 381 p.

POHL R.W. 1980. Flora costaricensis, family \#15 Gramineae. Field Museum of Natural History, Iowa State University, United States of America. 609 p.

RETANA J. 2011. Climatología de la región del distrito de Santa Rosa de Oreamuno en base a estaciones meteorológicas cercanas a la zona. Informe anual del Instituto Meteorológico Nacional. 3 p.

VAN SOEST P.J., ROBERTSON J.B. 1985. Analysis of forages and fibrous feeds. Cornell University. Ithaca, New York. 165 p.

VILLALOBOS L., SANCHEZ J. 2010a. Evaluación agronómica y nutricional del pasto ryegrass perenne tetraploide (Lolium perenne) producido en lecherías de las zonas altas de Costa Rica. I. Producción de biomasa y fenología. Agronomía Costarricense 34(1):31-42.

VILLALOBOS L., SANCHEZ J. 2010b. Evaluación agronómica y nutricional del pasto ryegrass perenne tetraploide (Lolium perenne) producido en lecherías de las zonas altas de Costa Rica. II. Valor nutricional. Agronomía Costarricense 34(1):43-52.

WEISS W. 2008. Review of Energy Nutrition: 2001 NRC Energy System. In: Curso de Nutrición de Ganado de Leche. Balsa de Atenas, Alajuela, Costa Rica.

WINGCHING R., ROJAS A. 2006. Nitrógeno orgánico y químico en sorgo negro con cobertura permanente de maní forrajero. II. Fraccionamiento de la proteína. Agronomía Costarricense 30(2):61-69. 
